\title{
Impact of Measurement Configurations on the Accuracy of Propagation Model Estimation with Applications to Dynamic Spectrum Access
}

\author{
Jad Nasreddine, Janne Riihijärvi, Petri Mähönen \\ Institute for Networked Systems, RWTH Aachen University \\ Kackertstrasse 9, D-52072 Aachen, Germany \\ email: $\{$ jad, jar, pma\}@inets.rwth-aachen.de
}

\begin{abstract}
In this paper we investigate the possibility of using measurement-based propagation models in the framework of Dynamic Spectrum Access (DSA), where secondary users can opportunistically access the licensed spectrum of a primary system. In principle this will provide a precise representation of the radio environment since it takes into account all environmental details at a given period of time. However the accuracy of the estimated models will depend on sensor density, propagation factors, and any external error. We study the impact of these factors on the accuracy of the estimated model and the satisfaction of primary and secondary networks. We also propose a guideline to overcome these problems in DSA scenarios by using a power margin.
\end{abstract}

\section{INTRODUCTION}

Dynamic spectrum access (DSA) has become an intensively studied approach for increasing the efficiency of spectrum use [1]-[3]. The use of cellular systems in the so-called TV white spaces (TVWS) has gained significant amount of interest recently [4], [5], and secondary access to other frequency bands has also been considered in the literature [6], [7].

Propagation models play a key role in many DSA related decisions. They are used in particular to estimate the interference experienced by a primary user for a given secondary transmitter configuration [8]-[12]. In the current regulation proposals regarding DSA [13], [14], propagation models are usually standardized, with either a single propagation model or a parametric family. These models are used for diverse environments on a country-wide scale. As shown in [15], any fixed propagation model can result in large estimation errors especially in typical urban environments, and hence can result in either excessive interference towards the primary or unnecessary loss of secondary capacity, depending on the type of the error. There is also some existing work on the estimation of customized propagation models [16], where propagation models are estimated based on local measurements. However, the impact of the different factors on the accuracy of such methods are still not thoroughly investigated. In this paper, we study the feasibility of a measurement-based approach for the determination of a customized propagation model, in which the secondary transceivers carry out geolocalized measurements of path losses. These measurements are then combined to form an estimate of the propagation model. In particular, we study in detail the accuracy with which propagation models could be estimated at the run-time of the system. We make a detailed numerical analysis on the number of measurement points needed for reaching a given level of accuracy, and we also study how additional impairments such as localization errors at measuring terminals influence the results. We give our results both in terms of error distributions of the different coefficients of a standard power law path loss model complemented with model of shadow fading, as well as in terms of the impact of these results on the interference probability in a DSA scenario. We also briefly discuss how the studied approach could be implemented in the context of existing wireless communication networks. It should be noted that the model can be also extended to any other systems that require accurate and localized path loss models such as the case of small cell scenario, but we limit our study in this paper to the case of DSA for TVWS, as an illustrative example.

The rest of this paper is structured as follows. In Section II we discuss our system model for path loss estimation in more detail. We then present the used evaluation scenarios for studying the impact of measurement configurations on the accuracy of path loss estimates within our model in Section III, and discuss our results in Section IV. Finally, we draw conclusions and discuss future work in Section V.

\section{System Model for PATh Loss Estimation}

Most of the work on TV white space assumes that the path loss model and its parameters are known. Other existing works use terrain-based propagation simulators to generate the path loss estimates. The problem with the first approach is the potentially large errors originating from the regions the propagation model is not well suited to. Although the second approach can provide accurate models, it requires very detailed information about the environment (e.g. information about trees, pedestrians, cars, in addition to buildings). The detailed information is very difficult to obtain especially for small obstacles. In addition, the generation of propagation models using terrain-based simulators with the aforementioned required level of details may necessitate unacceptable computational costs. Because of these issues we study in this paper a third alternative, a measurement-based approach. We assume that some of the transceivers in the secondary network have 
information on their locations, and are capable of carrying out measurements on the path loss between themselves and active secondary transmitters. The collection of information can be performed through, for instance, the Minimization of Drive-Tests (MDT) functionality, which is an LTE release 10 feature [17]-[19]. The measurement results can then be combined into path loss models to be used within the region. Our approach is closely related to the use of spatial interpolation techniques as discussed in [20].

We adopt here the classical power law model for the largescale distance-dependent path loss component combined with log-normal shadowing. That is, we assume that at distance $d$ the overall path loss in $\mathrm{dB}$ can be written as

$$
L(d)=k+\alpha \log d+\chi,
$$

where $k$ and $\alpha$ are constants to be determined, and $\chi$ is a zero-mean normal random variable with standard deviation $\sigma$, also to be determined. We assume fast fading to be averaged out during the measurement phase, and accordingly do not consider its impact in the following. This is done mainly for space reasons, as incorporating fast fading presents no fundamental difficulty, but would significantly increase the complexity of the expressions obtained below.

Now, given a collection of measured path loss values, the constants $k$ and $\alpha$ can be found by simple linear regression. We apply the ordinary least squares estimator for solving this regression problem. This results in estimates $k_{\text {est }}$ and $\alpha_{\text {est }}$ for the path loss coefficients. Provided that the model in (1) is accurate, the estimated values converge to true values $k$ and $\alpha$ when the number of measurement points becomes large enough. Once the distance-dependent part has been found, $\sigma$ can be estimated as the sample standard deviation of the residuals, that is, the differences between the measured values and the fitted distance dependent path loss model. We shall denote this estimate in the following by $\sigma_{\text {est }}$.

The key issue that we shall now study is how does the number and accuracy of the path loss measurements influence the accuracy of these estimates, and how large is the impact of any remaining inaccuracies in the context of DSA. As mentioned above, provided that the overall path loss model is correct, in the limit of large number of measurements high accuracy is to be expected. However, especially during the initialization phase of the system or due to the sparseness of the secondary network only very limited number of measurements might be available, resulting in potentially significant errors in the estimates. Further, these errors will depend on the shadowing process, the locations the measurements are carried out, and possible inaccuracies either in the measurement results themselves or in the location estimates.

\section{Evaluation Scenarios AND Metrics}

In this section, we shall describe the evaluation scenario and the used metrics. We consider a scenario in which a cellular network is acting as a secondary system using TV white space, as shown in 1. This scenario is chosen due to the crucial impact of path loss accuracy in this case. In order to enable spectrum

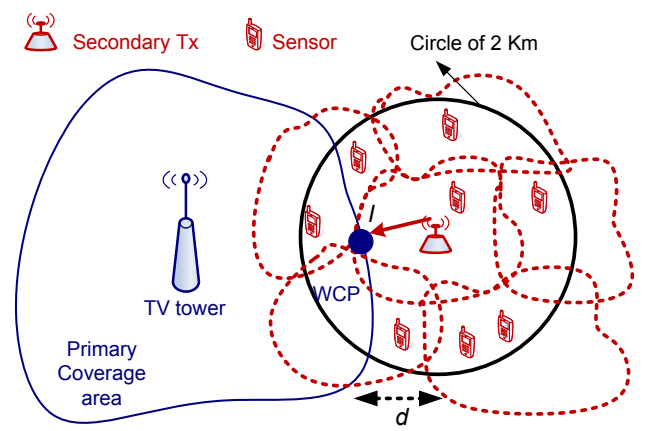

Fig. 1. An illustrative example of the considered scenario.

sharing we assume that the secondary network can access to a database where the positions and channels used by the TV towers can be found [21]. This work consider only downlink, and can be extended to uplink using the framework in [22]. We assume that the secondary network knows the positions of its own base stations. To protect primary network, we define interference probability $\mathrm{Pr}_{\text {int }}$ as the probability that a primary receiver experience an interference $I$ higher than a defined threshold $\iota_{\max }$ due to secondary activity [10], [11], [23]:

$$
\operatorname{Pr}_{\text {int }} \triangleq \mathbb{P}\left\{I \geq \iota_{\max }\right\} \text {. }
$$

This probability should be lower that a defined threshold $\varepsilon$ for all primary receivers. Since the latter have unknown positions, the Worst Case Position (WCP) is normally used [22], [24]. The WCP is defined as the closest position inside the primary coverage area to the secondary transmitter. In the case we have a distant-dependent path loss and a shadowing factor with constant standard deviation, respecting the interference constraint at the WCP means that this constraint is respected in all receiver positions as well. Hence, the secondary transmitter should estimate its allowed transmit power, $P_{S}$, taking into account the interference constraint considering the WCP. The first step is therefore to estimate the path loss model between its antenna and a receiver antenna at the WCP, for which the secondary transmitter knows the separating distance $d$. Then the determination of $P_{S}$ is straightforward when the propagation model is determined.

In our model, the secondary receivers are spread over a large area and can decode the signal of the base stations, which is the case of any cellular network. Hence, these receivers can estimate the path loss model with some errors as explained in the previous section. By considering (2) and assuming a log-normal shadowing factor, the secondary can estimate its transmit power, in the second step, using

$$
P_{S}=\iota_{\max }-\sqrt{2} \sigma_{\text {est }} \mathrm{erf}^{-1}(1-2 \varepsilon)+k_{\mathrm{est}}+\alpha_{\mathrm{est}} \log _{10}(d) .
$$

We assume in this paper that the secondary receiver positions are uniformly distributed inside a circle of $2 \mathrm{~km}$ radius centered at the secondary transmitter (i.e. base station), and the WCP is at a distance $d$ from the latter; we assume further that distance $d$ is lower than $2 \mathrm{~km}$ so that the WCP is covered by the measurement sensors. The $2 \mathrm{~km}$ circle is not the coverage area 
of the considered transmitter but it can include the coverage area of several transmitters that belong to the same operator. We also assume that the path loss has a distance-dependent factor that follows the Okumura-Hata model [25], [26], and a Gaussian shadowing factor (in the logarithmic scale) with standard deviation $\sigma$. The performance evaluation is made in an urban zone using a frequency of $700 \mathrm{MHz}$, where the base station antenna effective height is $30 \mathrm{~m}$ and the secondary receiver antenna height is $h$. The primary receiver is either at $1.5 \mathrm{~m}$ or $10 \mathrm{~m}$ to show the difference between the cases where the primary antenna is at floor level or at roof level. The system performance is evaluated through several metrics:

- The distribution of the error percentage in the estimated path loss exponent $\left(e_{\alpha}\right)$, path loss constant $\left(e_{k}\right)$, and shadowing factor standard deviation $\left(e_{\sigma}\right)$, given by

$$
e_{X}=100 \frac{\left(X_{\mathrm{est}}-X\right)}{X},
$$

where $X$ stands for any of the evaluated factors (i.e. $\alpha, k$, and $\sigma$ ), and $X_{\text {est }}$ is the estimated value of these factors.

- The distribution of the interference probability experienced by a primary receiver at distance $d$ from the secondary transmitter. Assuming a normal distribution of the shadowing factor, and using (2) and (3), we can write

$$
\begin{aligned}
\operatorname{Pr}_{\text {int }}=\frac{1}{2}[1+\operatorname{erf}( & \frac{k_{\text {est }}-k+\left(\alpha_{\text {est }}-\alpha\right) \log _{10}(d)}{\sqrt{2} \sigma} \\
& \left.\left.-\frac{\sigma_{\text {est }}}{\sigma} \operatorname{erf}^{-1}(1-2 \varepsilon)\right)\right] .
\end{aligned}
$$

The performance of the path loss and power estimators is affected by the presence of different errors generated by different sources. In this paper we study the impact of the following parameters that induce errors in the estimators:

- Sensor density: It influences directly the estimation of the path loss parameters, especially $\alpha$ and $k$. We consider that the density is in the interval $[20,200]$ sensors $/ \mathrm{km}^{2}$, which is a typical interval in cellular networks.

- Shadowing factor standard deviation: As can be seen from (5), this factor has a direct impact on the interference probability. It impacts also the estimation of the path loss exponent and constant.

- Noise power: This includes all errors related to the measurement hardware such as errors in the noise power, additional interference from far away transmitters of the same system, or antenna gains. Herein, we assume that these errors follows (in the logarithmic scale) a normal distribution, as it is usually considered in the field, with zero mean and a standard deviation of $1 \mathrm{~dB}$.

- Positioning error: All positioning mechanisms estimate the location with certain precision. Therefore, we assume that the sensors have an positioning error that is uniformly distributed in the interval $[-15,15] \mathrm{m}$.

- Wall penetration losses: The secondary sensors can be outdoor or indoor. In the latter case, several walls can separate the sensor from the transmitter and induce some penetration losses. In this paper we assume that the number of blocking walls can be 0,1 , or 2 , and that the penetration loss of one wall is $10 \mathrm{~dB}$. Hence, we consider an additional received power with a discrete uniform distribution on the integers 0,10 , and $20 \mathrm{~dB}$.

- Sensor antenna height: The secondary sensors can also have different antenna heights $h_{r}$ since they may be at different floors of a building, for instance. The difference in the antenna height will lead to additional variation in the path loss. The receiver antenna height is included in the path loss model for medium-small cities through the following formula [26]:

$$
h_{e}=\left[1.1 \log _{10}(f)-0.7\right] h_{r}-1.56 \log _{10}(f)+0.8 \text {, }
$$

where $f$ is the central frequency, which is considered to be $700 \mathrm{MHz}$. For this frequency and for antenna height between $1.5 \mathrm{~m}$ and $10 \mathrm{~m}$, a difference of $20 \mathrm{~dB}$ can be observed. Therefore we consider an additional power that follows a uniform distribution in the interval $[0,20] \mathrm{dB}^{1}$.

\section{RESULTS}

We have evaluated the performance of the path loss and transmit power estimators under several conditions mapped into three scenarios:

- Scenario 1: We assume that there is no error in the measurements reported by the sensors.

- Scenario 2: We consider only positioning errors.

- Scenario 3: We consider positioning, noise, antenna height, and wall penetration loss errors.

For all scenarios we generated $10^{4}$ samples with different sensor positions, shadowing factor, and errors, after which we collected the generated statistics considering a target interference probability $\varepsilon=0.05$.

We shall first study the impact of the sensor density and the shadowing standard deviation on the estimators in scenario 1 . In Fig. 2, we show the impact of sensor density and shadowing standard deviation on the distribution of the error percentage and the interference probability when the primary receiver antenna height is $1.5 \mathrm{~m}$ and the distance separating the primary receiver and secondary transmitter $d$ is $1.5 \mathrm{~km}$. We can first notice that the errors are unbiased (i.e. the error percentages have zero mean while the interference probabilities have a mean equal to $\varepsilon$ ). The figures show also that the variances of the errors in the path loss exponent and constant are an increasing function of the shadowing factor standard deviation, but the latter has insignificant impact on the error on the standard deviation. Although the interference probability, as it can be seen from (5), depends on the errors in the path loss constant and exponent that are affected by the standard deviation, the latter does not have high impact on the interference probability. This is due to the fact that the error increases in the exponent and the constant are compensated by the increase of the value of the standard deviation as it can be seen in (5). For high sensor density, the errors in the path loss factors do not exceed

\footnotetext{
${ }^{1}$ For large city, the interval will be $[0,4] \mathrm{dB}$.
} 


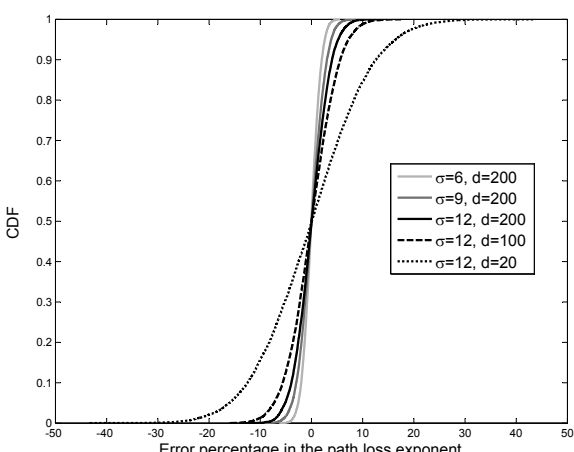

(a)

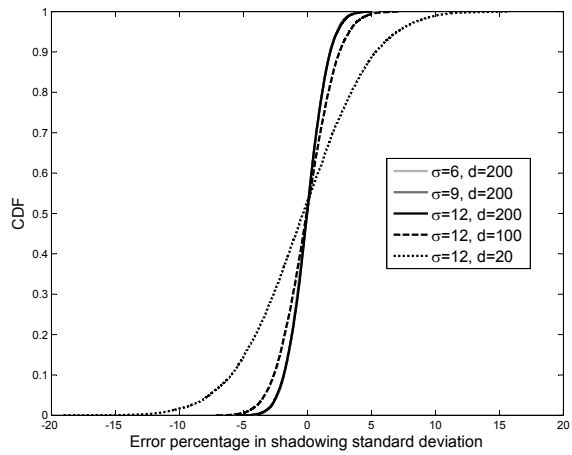

(c)

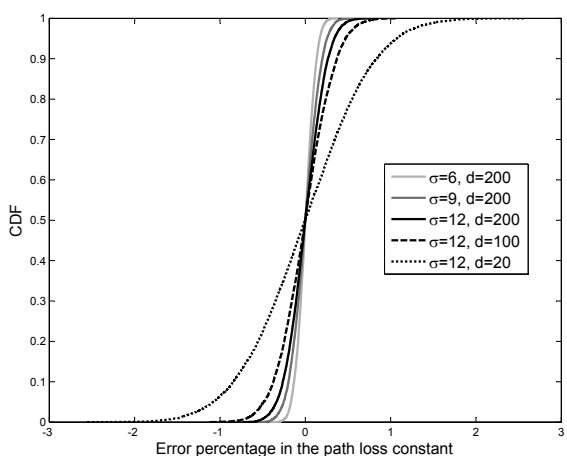

(b)

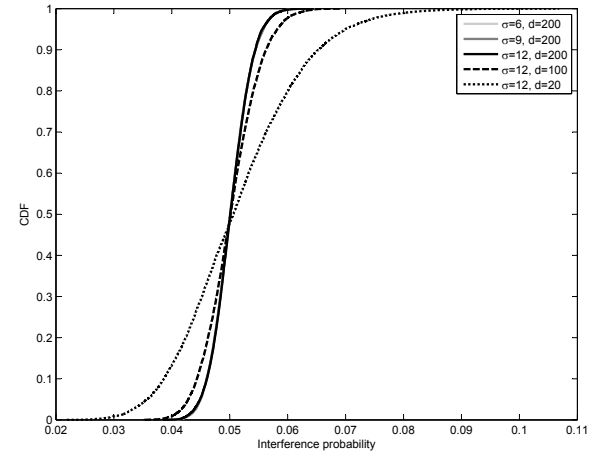

(d)

Fig. 2. The distribution of the error percentages and interference probability for different values of sensor density and shadowing standard deviation in scenario 1 , when primary antenna height is $1.5 \mathrm{~m}$.

few percents and the interference probability is always lower than 0.6. However, the error can reach more than $20 \%$ in the path loss exponent when the density of the sensors is around 20 sensors $/ \mathrm{km}^{2}$. This leads to high interference probability that exceeds 0.1. These results show that although the average interference probability respects primary constraint in the case where no errors are considered, the interference probability can reach high value, which cannot be tolerated by the primary system. Hence, it is very important to take into account the distribution of the interference probability and not only its average, as considered by most of the studies.

In Fig. 3, we show the distribution of the interference probability when primary antenna height is $10 \mathrm{~m}$, which represents the worst case for TV receivers in operational networks when the antenna is at the roof of a building. The figure shows, clearly, the high impact of the difference in antenna heights on the interference probability. This is due to the $20 \mathrm{~dB}$ difference that appears in the estimated path loss constant $k$ due the difference between the antenna heights of the primary receivers and the secondary sensors. Moreover, the value of $\sigma$ has very high impact, since the factor including the error in the path loss constant is the influential factor in this case. It should be noted that the interference probability can reach more than 0.95 for a standard deviation of $6 \mathrm{~dB}$.

We have also evaluated the impact of positioning errors in scenario 2. Our results (not shown here for simplicity) indicate that the impact of these errors is very low, due to

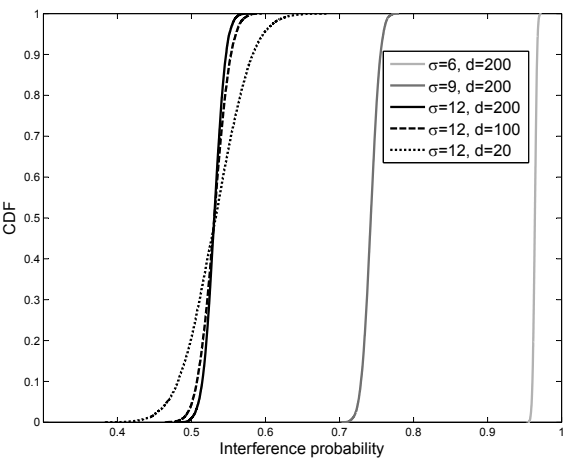

Fig. 3. The distribution of the interference probability for different values of sensor density and shadowing standard deviation in scenario 1 , when primary antenna height is $10 \mathrm{~m}$.

the small error considered in positioning systems. These errors correspond to the ones obtained in GPS systems for outdoor environments. In case we assume lower precision the impact is expected to be higher. This study is left for future work.

In scenario 3, we have studied the impact of the different measurement errors considering the TV receiver antenna is at $10 \mathrm{~m}$. The results are depicted in Fig. 4 and show how these errors impact system performance. The high impact appear clearly in the intolerable errors in $k, \sigma$ and the generated probability of interference. The estimation of the path loss ex- 


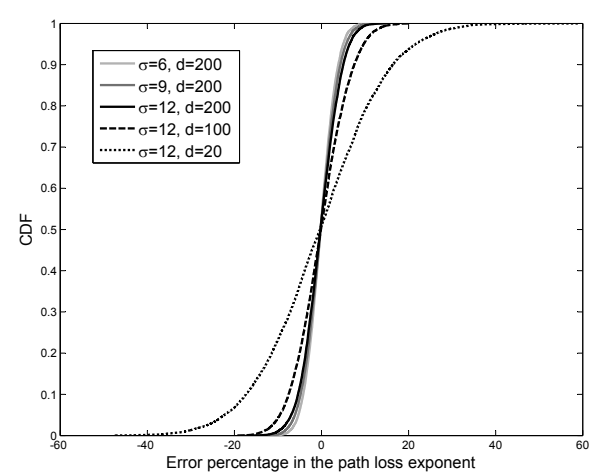

(a)

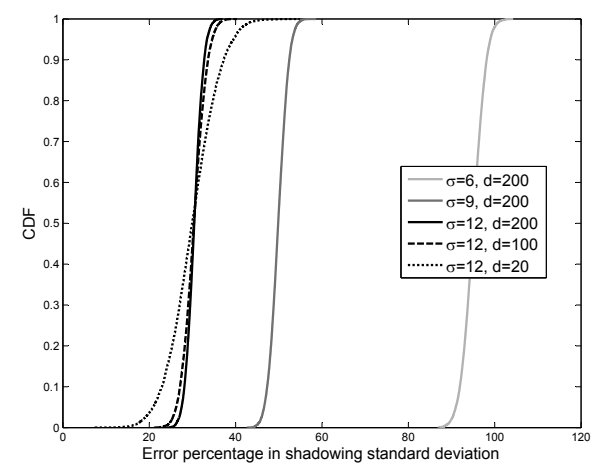

(c)

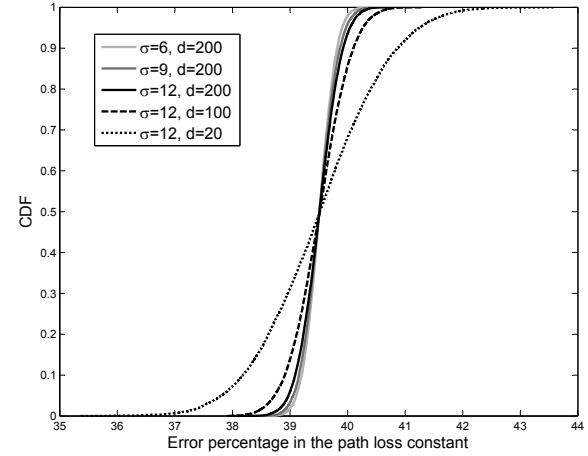

(b)

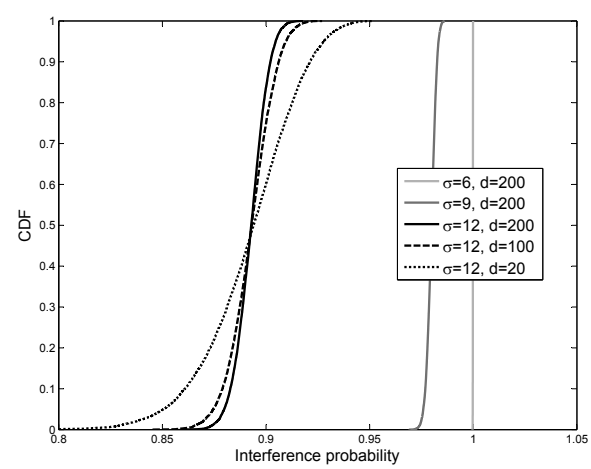

(d)

Fig. 4. The distribution of the error percentages and interference probability for different values of sensor density and shadowing standard deviation in scenario 3 .

ponent is not significantly affected since, with the exception of the positioning error, all errors are additive in the logarithmic domain. This is reflected by the fact that the mean error is shifted by a significant percentage. It is also interesting that these errors have also moved the average error in the estimated shadowing standard deviation, where high errors that can reach the double of the real standard deviation can be observed for low $\sigma$. This is due to the additional stochastic parameters (i.e. the additive errors) that have a total standard deviation of $10.2 \mathrm{~dB}$, as our numerical estimation showed. This makes the total standard deviation of the measured power to vary between $11.8 \mathrm{~dB}$ and $15.7 \mathrm{~dB}$ when the real standard deviation varies between $6 \mathrm{~dB}$ and $12 \mathrm{~dB}$.

As it was shown in the results, the interference probability can be very different depending on the sensor configurations. Since the average value of the interference probability does not provide comprehensive information about system performance, the primary network would prefer a stronger metric that guarantee the QoS for its users. Therefore, a better metric can be the $95^{\text {th }}$ percentile of the probability of interference, which guarantee that in $95 \%$ of the cases, the interference probability will be less than $\varepsilon$. In addition and as it can be seen from (5), the interference probability depends on distance $d$. In Fig. 5 we show the $95^{\text {th }}$ percentile of the interference probability as a function of the distance in the two study cases of scenario 1 , and in scenario 3 .
To comply with primary constraint, the secondary network should extract from $P_{S}$ in (3) a power margin $M$, which can be computed for each sample by combining (3) and (5):

$$
\begin{aligned}
M= & k_{\text {est }}-k+\left(\alpha_{\text {est }}-\alpha\right) \log _{10}(d) \\
& -\sqrt{2}\left(\sigma_{\text {est }}-\sigma\right) \operatorname{erf}^{-1}(1-2 \varepsilon) .
\end{aligned}
$$

The $95^{\text {th }}$ percentile of the margin given in (7) is depicted in Fig. 6 for the different scenarios. We use the $95^{\text {th }}$ percentile to guarantee that in $95 \%$ of the cases the interference probability is satisfied. This margin reflects the impact of the estimation errors on secondary network performance; when high margin is required, the allowed transmit power will be lower and thus the secondary performance will decrease. Fig. 6 shows that the required margin can be less than $4 \mathrm{~dB}$ for a distance less than $2 \mathrm{~km}$ if there is no additional errors. However this margin can reach up to $30 \mathrm{~dB}$ if all errors are considered. In addition, the required power margin is not a monotonic function of the distance and it has a minimum around $1.2 \mathrm{~km}$ in this case. A more interesting result is the fact that, although for low $\sigma$ the $95^{\text {th }}$ percentile of the interference probability is very high, the power margin is very low. This is a direct outcome of (5) and (7), where the first shows that the interference probability is inversely proportional to $\sigma$ while the second shows that the margin depends only on the error on $\sigma$. This is very important since it confirms the known fact that the interference probability can be very high, while the interference values 


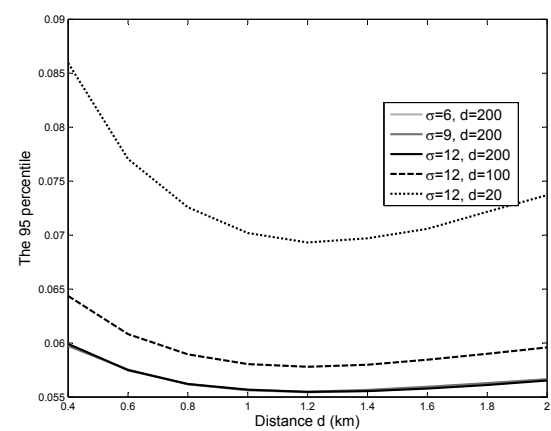

(a)

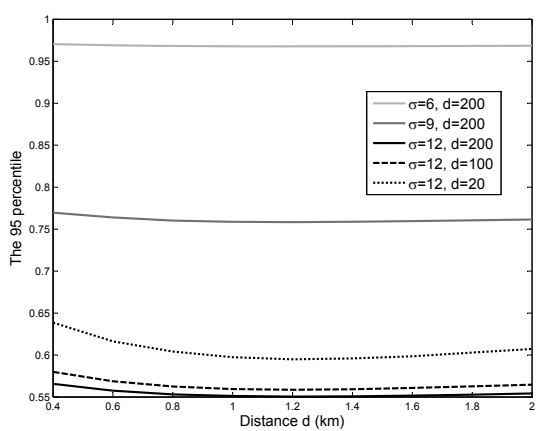

(b)

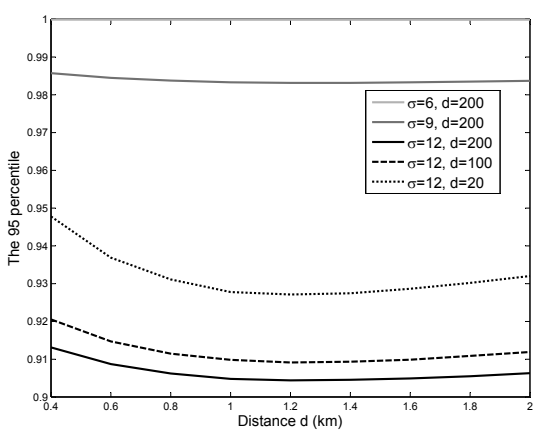

(c)

Fig. 5. The $95^{\text {th }}$ percentile of the interference probability as a function of the distance $d$ for (a) scenario 1 when primary antenna height is $1.5 \mathrm{~m}$, (b) scenario 1 when primary antenna height is $10 \mathrm{~m}$, and (c) scenario 3 when primary antenna height is $10 \mathrm{~m}$.

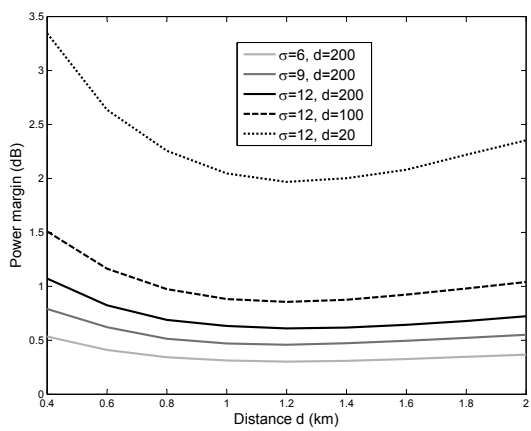

(a)

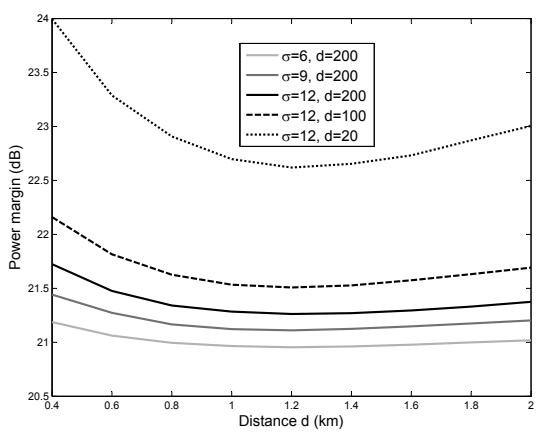

(b)

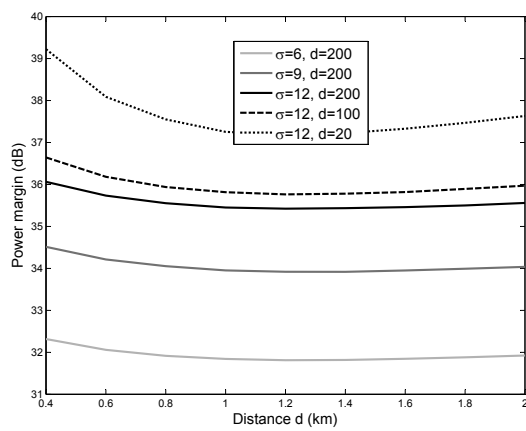

(c)

Fig. 6. The power margin that should be extracted from the estimated power to guarantee that the $95^{\text {th }}$ percentile of the interference probability is lower than 0.05 for (a) scenario 1 when the primary antenna height is $1.5 \mathrm{~m}$, (b) scenario 1 when the primary antenna height is $10 \mathrm{~m}$, and (c) scenario 3 when the primary antenna height is $10 \mathrm{~m}$.

are very close to the specified threshold when the standard deviation is small enough. It should be noted that the margin is computed assuming that the errors are known in each sample, which makes these results a lower bound for any realistic margin.

\section{CONClusions AND Discussion}

In this paper we have studied the possibility of estimating path loss models for functional networks, the impact of different factors on the accuracy of the estimation, and the impact of the latter on the performance of Dynamic Spectrum Access (DSA). We have also studied the required margin that should be extracted from the estimated power by the secondary network in case of estimation errors. Based on our results, we can draw the following conclusions:

- Sensor density has a paramount impact on all propagation components (i.e. path loss constant and exponent, and standard deviation of the shadowing factor), interference probability, and required power margin.

- The real standard deviation of the shadowing factor has low impact on the propagation constant and exponent, and no impact on the shadowing standard deviation and interference probability, when no external errors are present in the system. However it has always a significant impact on the power margin, especially when all errors are considered. Moreover the impact of this factor on the path loss exponent and constant does not change significantly when the external errors are considered, but it drastically increases the errors of the standard deviation and interference probability in this case.

- The external errors do not impact the propagation exponent but has a crucial impact on the other factors. This is logical since the considered errors are additive. The difference in the antenna heights, which has a high impact, can be compensated by a protection factor if we know the distribution of the antenna heights.

- The power margin can be estimated if the distributions of the errors in the propagation factors are known. The determination of these distributions is a very interesting problem and depends on the scenario. This paves the way to develop secondary networks with reliable guarantees on respecting primary constraints.

- The power margin does not change too much with the distance (i.e. only couple of $\mathrm{dBs}$ ) but it is highly depending on the external errors, the real standard deviation of the shadowing factor, and the sensor density. It is an increasing function of the standard deviation and, as expected, decreases when the sensor density increases. 
- In existing works, the interference probability has been considered as a metric to protect primary systems. However, when dynamic estimation or propagation models are considered the interference probability will depend on the distribution of the errors, and hence this probability will have itself a distribution. In addition, using the average values of the errors and interference probability does not provide the necessary information about system performance. For instance, the estimated propagation constants and interference probability can be the same as the real ones in average, but they might have high errors in some cases. Therefore we argue that higher moments or/and given percentiles of the interference probability should be considered instead of the average.

- The current regulation for TV white space does not provide good mechanisms to protect primary systems, especially in urban areas where the simplistic propagation models may lead to significant errors in interference estimation. Our study can be used as ground base for measurement-based estimation of the path loss models that can be used for interference assessment when the traditional models are not suitable. Measurement-based models should be then required by regulators to allow opportunistic access. More specifically, the secondary should report their measurements and the characteristics of their sensors to primary networks or regulators. Acquiring this type of information will not be a problem in future networks especially when the minimization of drive-tests is deployed [17], [18].

Based on this work, we are now developing analytical and numerical methods to determine the error distributions in the estimated factors using standard deviation distribution. We are also considering the case when there is a structural mismatch between the considered model and the real propagation model, which can be different than Okumura-Hata model. A model will be also developed for the case where secondary sensors are not uniformly distributed and with correlated shadowing.

\section{ACKNOWLEDGMENT}

The authors would like to thank RWTH Aachen University and the German Research Foundation (Deutsche Forschungsgemeinschaft, DFG) for providing financial support through the UMIC research centre. We would also like to thank the European Union for providing partial funding of this work through the FARAMIR project.

\section{REFERENCES}

[1] Q. Zhao and B. Sadler, "A survey of dynamic spectrum access," IEEE Signal Processing Magazine, vol. 24, no. 3, pp. 79 -89, May 2007.

[2] S. Geirhofer, L. Tong, and B. Sadler, "Cognitive radios for dynamic spectrum access - dynamic spectrum access in the time domain: Modeling and exploiting white space," IEEE Communications Magazine, vol. 45 , no. 5, pp. $66-72$, May 2007.

[3] Y.-C. Liang, K.-C. Chen, G. Li, and P. Mähönen, "Cognitive radio networking and communications: An overview," IEEE Transactions on Vehicular Technology, vol. 60, no. 7, pp. 3386 -3407, Sept. 2011.
[4] J. Wang, M. Ghosh, and K. Challapali, "Emerging cognitive radio applications: A survey," IEEE Communications Magazine, vol. 49, no. 3, pp. $74-81$, March 2011.

[5] Y.-J. Choi and K. Shin, "Opportunistic access of tv spectrum using cognitive-radio-enabled cellular networks," IEEE Transactions on Vehicular Technology, vol. 60, no. 8, pp. 3853 -3864, Oct. 2011.

[6] J. Nasreddine, A. Achtzehn, J. Riihijärvi, and P. Mähönen, "Enabling secondary access through robust primary user channel assignment," in IEEE Global Telecommunications Conference (GLOBECOM 2010), Miami, FL., USA, Dec. 2010.

[7] R. Saruthirathanaworakun, J. Peha, and L. Correia, "Opportunistic primary-secondary spectrum sharing with a rotating radar," in International Conference on Computing, Networking and Communications (ICNC 2012), Maui, Hawaii, Feb. 2012, pp. 1025 -1030.

[8] X. Hong, C.-X. Wang, and J. Thompson, "Interference modeling of cognitive radio networks," in IEEE Vehicular Technology Conference (VTC Spring 2008), Singapore, May 2008, pp. $1851-1855$.

[9] R. Tandra, M. Mishra, and A. Sahai, "What is a spectrum hole and what does it take to recognize one?" Proceedings of the IEEE, vol. 97, no. 5, pp. 824-848, May 2009.

[10] B. L. Mark and A. O. Nasif, "Estimation of maximum interference-free power level for opportunistic spectrum access," IEEE Transactions on Wireless Communications, vol. 8, no. 5, pp. 2505 -2513, May 2009.

[11] J. Nasreddine, J. Riihijärvi, and P. Mähönen, "Location-based adaptive detection threshold for dynamic spectrum access," in the 4th IEEE International Symposium on New Frontiers in Dynamic Spectrum Access Networks (DySPAN 2010), Singapore, April 2010.

[12] _ - "Transmit power control for secondary use in environments with correlated shadowing," in IEEE International Conference on Communications (ICC 2011), Kyoto, Japan, July 2011.

[13] FCC, "In the matter of unlicensed operation in the TV broadcast bands: Second memorandum opinion and order," Tech. Rep. 10-174A1, 2010.

[14] ECO WG SE43, "ECC Report 159: Technical and operational requirements for the possible operation of cognitive radio systems in the white spaces of the frequency band 470-790 MHz," 2011.

[15] C. Phillips, D. Sicker, and D. Grunwald, "Bounding the error of path loss models," in the 5th IEEE Symposium on New Frontiers in Dynamic Spectrum Access Networks (DySPAN 2011), Aachen, Germany, May 2011, pp. $71-82$.

[16] A. Alaya-Feki, A. L. Cornec, and E. Moulines, "Optimization of radio measurements exploitation in wireless mobile networks," Journal of communications (JCM), vol. 2, no. 7, pp. 59-67, 2007.

[17] 3GPP TS 37.320 v11.0.0, "Radio measurement collection for Minimization of Drive Tests (MDT); overall description; stage 2," June 2012.

[18] 3GPP TR 36.805 v9.0.0, "Study on Minimization of drive tests in Next Generation Networks." Dec. 2010.

[19] T. Cai, J. van de Beek, B. Sayrac, S. Grimoud, J. Nasreddine, J. Riihijärvi, and P. Mähönen, "Design of layered radio environment maps for RAN optimization in heterogeneous LTE systems," in IEEE 22nd International Symposium on Personal Indoor and Mobile Radio Communications (PIMRC 2011), Toronto, Canada, Sept. 2011, pp. 172 -176.

[20] B. Sayrac, J. Riihijärvi, P. Mähönen, S. Jemaa, E. Moulines, and S. Grimoud, "Imporving coverage estimation for cellular networks with spatial bayesian prediction based on measurements," in ACM CellNet 2012 in conj. with ACM SIGCOMM 2012, Helsinki, Finland, Aug. 2012.

[21] R. Murty, R. Chandra, T. Moscibroda, and V. Bahl, "Senseless: A database-driven white spaces network," IEEE Transactions on Mobile Computing, vol. 11, no. 2, pp. 189 -203, Feb. 2012.

[22] J. Nasreddine, O. Sallent, J. Pérez-Romero, and R. Agustí, "Positioningbased framework for secondary spectrum usage," Physical Communication, vol. 1, no. 2, pp. 121-133, 2008.

[23] A. Ghasemi and E. Sousa, "Interference aggregation in spectrum-sensing cognitive wireless networks," IEEE Journal of Selected Topics in Signal Processing, vol. 2, no. 1, pp. 41 -56, Feb. 2008.

[24] M. Pirmoradian and C. Politis, "Cumulative interference," IEEE Vehicular Technology Magazine, vol. 5, no. 3, pp. 39 -45, Sept. 2010.

[25] Y. Okumura, E. Ohmori, T. Kawano, and K. Fukuda, "Field strength and its variability in VHF and UHF land-mobile radio service," Rev. Elec. Commun. Lab., vol. 16, pp. 825-873, Sept. 1968.

[26] M. Hata, "Empirical formula for propagation loss in land mobile radio service," IEEE Trans. Veh. Technol., vol. VT-29, pp. 317-325, 1980. 